\title{
Ethnic and sex differences in hepatic lipid content and related cardiometabolic parameters in lean individuals
}

\author{
Kitt Falk Petersen, ${ }^{1,2,3}$ Sylvie Dufour, ${ }^{1,2}$ Fangyong Li, ${ }^{4}$ Douglas L. Rothman, ${ }^{5,6}$ \\ and Gerald I. Shulman ${ }^{1,2,7}$ \\ 'Department of Internal Medicine and ${ }^{2}$ Yale Diabetes Research Center, Yale School of Medicine, New Haven, Connecticut, \\ USA. ${ }^{3}$ Novo Nordisk Foundation Center for Basic Metabolic Research, University of Copenhagen, Copenhagen, Denmark. \\ ${ }^{4}$ Yale Center for Analytical Sciences, Yale School of Public Health, New Haven, Connecticut, USA. ${ }^{5}$ Department of Radiology \\ and Biomedical Imaging, Yale School of Medicine, New Haven, Connecticut, USA. ${ }^{6}$ Department of Biomedical Engineering, \\ Yale School of Engineering and Applied Science, New Haven, Connecticut, USA. 'Department of Cellular and Molecular \\ Physiology, Yale School of Medicine, New Haven, Connecticut, USA.
}

Conflict of interest: GIS is an inventor on Yale patents for livertargeted mitochondrial uncoupling agents and controlled-release mitochondrial uncoupling agents for the treatment of nonalcoholic fatty liver disease, nonalcoholic steatohepatitis, type 2 diabetes, and related metabolic disorders (title: "Novel 2,4-Dinitrophenol Formulations and Methods Using Same"; patent application numbers: 61/872,294, 61/919,003, 14/911,322, and 14/911,322; patent number: $10,786,466)$ and is a scientific cofounder of TLC Inc. GIS serves on the advisory boards for Merck, Novo Nordisk, AstraZeneca, Aegerion, Levels, Bayer, Equator Therapeutics, Generian, and Janssen Research and Development. GIS receives investigator-initiated support from AstraZeneca, Gilead Sciences, Merck, and Maze Therapeutics.

Copyright: (c) 2022, Petersen et al. This is an open access article published under the terms of the Creative Commons Attribution 4.0 International License.

Submitted: December 23, 2021

Accepted: February 9, 2022

Published: April 8, 2022

Reference information: JCI Insight 2022;7(7):e157906.

https://doi.org/10.1172/jci.

insight.157906.
BACKGROUND. Nonalcoholic fatty liver affects $25 \%$ to $30 \%$ of the US and European populations; is associated with insulin resistance (IR), type 2 diabetes, and increased cardiovascular risk; and is defined by hepatic triglyceride (HTC) content greater than $\mathbf{5 . 5 6 \%}$. However, it is unknown whether HTC content less than $\mathbf{5 . 5 6 \%}$ is associated with cardiometabolic risk factors and whether there are ethnic (Asian Indian, Al, versus non-AI) and/or sex differences in these parameters in lean individuals.

METHODS. We prospectively recruited 2331 individuals and measured $\mathrm{HTC}$, using ${ }^{1} \mathrm{H}$ magnetic resonance spectroscopy, and plasma concentrations of triglycerides, total cholesterol, LDLcholesterol, HDL-cholesterol, and uric acid. Insulin sensitivity was assessed using Homeostatic Model Assessment of Insulin Resistance and the Matsuda Insulin Sensitivity Index.

RESULTS. The 95th percentile for HTC in lean non-Al individuals was $1.85 \%$. Plasma insulin, triglycerides, total cholesterol, LDL-cholesterol, and uric acid concentrations were increased and HDL-cholesterol was decreased in individuals with HTC content $>1.85 \%$ and $\leq 5.56 \%$ compared with those individuals with HTC content $\leq 1.85 \%$, and these altered parameters were associated with increased IR. Mean HTC was lower in lean non-AI women compared with lean non-AI men, whereas lean Al men and women had a $40 \%$ to $100 \%$ increase in HTC when compared with non-Al men and women, which was associated with increased cardiometabolic risk factors.

CONCLUSION. We found that the 95th percentile of HTC in lean non-Al individuals was $1.85 \%$ and that HTC concentrations above this threshold were associated with IR and cardiovascular risk factors. Premenopausal women were protected from these changes whereas young, lean AI men and women manifested increased HTC content and associated cardiometabolic risk factors.

FUNDING. Grants from the United States Department of Health and Human Resources (NIH/ National Institute of Diabetes and Digestive and Kidney Diseases): R01 DK113984, P30 DK45735, U24 DK59635, and UL1 RR024139; and the Novo Nordisk Foundation (NNF18CC0034900).

\section{Introduction}

Nonalcoholic fatty liver (NAFL) is a major predisposing factor for the cardiometabolic syndrome (1-4), type 2 diabetes (T2D; refs. 5-7), nonalcoholic steatohepatitis (NASH; ref. 8), cirrhosis (9), and hepatocellular carcinoma (10-13). The gold standard for noninvasively assessing hepatic triglyceride (HTG) content is localized ${ }^{1} \mathrm{H}$ magnetic resonance spectroscopy (MRS), and studies using this method or magnetic resonance imaging methods have demonstrated that NAFL affects between $20 \%$ and $30 \%$ of the US and European populations and can exist without abnormalities in markers of liver health such as liver enzymes (14-16). 
In this regard the current diagnostic criterion for NAFL is based on the Dallas Heart Study, in which HTG content was measured using ${ }^{1} \mathrm{H}$ MRS in a modified $1.5 \mathrm{~T}$ whole-body system in 345 individuals with a normal $\left(<25 \mathrm{~kg} / \mathrm{m}^{2}\right)$ BMI (15). In this study Szczepaniak et al. reported that the distribution of HTG content had a median HTG content of $1.9 \%$ and a 95 th percentile of $5.56 \%$ for the upper limit. Using this 95 th percentile as the cutoff to define NAFL, the authors found that $30.7 \%$ of 2287 individuals had NAFL and that NAFL was more prevalent in men versus women, which they attributed to increased alcohol consumption in the men (17). However, most of their participants were overweight $(33 \%)$ or obese $(43 \%)$ and had a history of moderate to excessive alcohol consumption $(\sim 70 \%)$ and/or diabetes $(\sim 10 \%)$. Previous studies by our group and others have suggested that normal HTG content in lean individuals is below 3\% $(16,18)$. Given that NAFL is found in lean individuals, even at the current definition of HTG greater than 5.56\%, the questions of whether even lower HTG content is associated with cardiometabolic risks and insulin resistance (IR) and whether there are ethnic and/or sex differences in these parameters in lean individuals remain.

To examine these questions, we prospectively recruited 2331 mostly young, lean, healthy individuals and measured HTG, intramyocellular lipid (IMCL), and extramyocellular lipid (EMCL) content using localized ${ }^{1} \mathrm{H}$ MRS. We performed a comprehensive metabolic characterization of each participant, including measuring concentrations of fasting plasma glucose, insulin, total cholesterol, triglycerides, HDL, LDL, aspartate aminotransferase (AST), alanine aminotransferase (ALT), and uric acid. In addition, we assessed IR in the overnight-fasted state, using the Homeostatic Model Assessment of Insulin Resistance (HOMA-IR; ref. 19), and in the postprandial state by performing oral glucose tolerance tests (OGTTs) to calculate the Matsuda Insulin Sensitivity Index (ISI; refs. 16, 20).

\section{Results}

Non-Asian Indian (non-AI) individuals were analyzed separately from the AI individuals, since, in earlier studies, we observed that the AI individuals as a group had a higher mean HTG compared with BMImatched non-AI individuals (ref. 16 and Figure 1). We found that the distribution for HTG content in all these lean non-AI individuals (BMI $<25 \mathrm{~kg} / \mathrm{m}^{2}$ ) was skewed to the right and had a median concentration of $0.35 \%$ and a 95 th percentile of $1.85 \%$ (Figure 2). In order to determine whether HTG $>1.85 \%$ has any cardiometabolic related significance as compared with the current criterion of HTG $>5.56 \%$, we compared fasting plasma markers of the cardiometabolic syndrome and IR between non-AI individuals with HTG $\leq$ $1.85 \%$ and with HTG content $>1.85 \%$ and $\leq 5.56 \%$ after adjusting for age, BMI, and sex. We found that fasting plasma concentrations of insulin, triglycerides, total cholesterol, LDL, and uric acid were all increased, and HDL was decreased in the individuals with HTG content $>1.85 \%$ and $\leq 5.56 \%$ compared with those individuals with HTG content $\leq 1.85 \%$ (Table 1 ). This group with HTG content $>1.85 \%$ and $\leq 5.56 \%$ also manifested increased diastolic blood pressure and whole-body IR as reflected by increased HOMA-IR and lower ISI compared with those with HTG content $\leq 1.85 \%$. Similar differences in these parameters were also observed in lean individuals (BMI $<25 \mathrm{~kg} / \mathrm{m}^{2}$ ) with $\mathrm{HTG}$ content $>1.85 \%$ and $\leq 5.56 \%$ compared with lean individuals with HTG content $\leq 1.85 \%$ (Supplemental Table 1; supplemental material available online with this article; https://doi.org/10.1172/jci.insight.157906DS1). Taken together these results demonstrate that HTG content $>1.85 \%$ and $\leq 5.56 \%$ is associated with IR and other risk factors for cardiometabolic disease.

To determine whether there are sex differences in HTG content and associated cardiometabolic parameters in non-AI individuals, we next compared these same parameters in men and women. We found that HTG content was significantly lower in women compared with men after adjusting for age and BMI and that this was associated with lower plasma concentrations of glucose, total cholesterol, LDL-cholesterol, and uric acid and increased concentrations of plasma HDL-cholesterol (Table 2). These changes were also associated with lower systolic and diastolic blood pressure in the women compared with the men.

Consistent with our prior study, we found that the mean HTG content was higher in the lean AI men compared with the lean non-AI men (Table 3 and ref. 16). Furthermore, this increased HTG in the lean AI men compared with the non-AI men was associated with increased fasting plasma glucose, insulin, and triglyceride concentrations; decreased HDL-cholesterol concentrations; and whole-body IR as reflected by increased HOMA-IR and decreased ISI (Table 3). Increased HTG content and IR in the lean AI men was also associated with increased IMCL and EMCL content as well as increased percentage of body fat.

We next examined whether in the AI population there are sex differences in HTG content and these same cardiometabolic parameters. Similar to the sex differences that we observed in non-AI individuals, we also observed an approximately $50 \%$ reduction of HTG in lean AI women compared with lean 


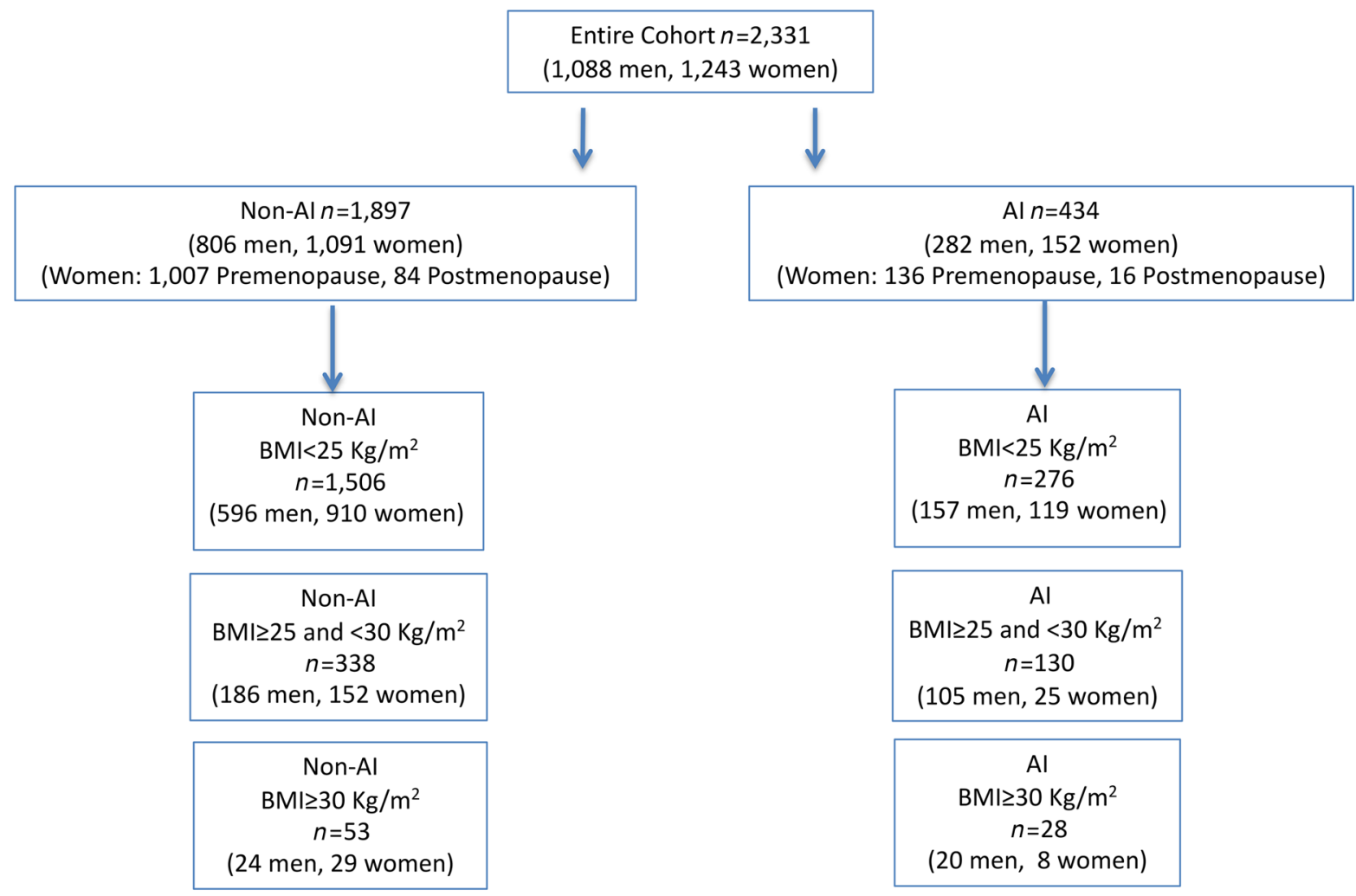

Figure 1. Flow diagram of recruited individuals and subgroups.

AI men (Supplemental Table 2), and this was associated with lower fasting plasma concentrations of glucose, triglycerides, and uric acid and increased plasma concentrations of HDL-cholesterol (Supplemental Table 2). These changes were also associated with reduced systolic and diastolic blood pressure in the AI women compared with the AI men.

Ethnic differences in HTG and these associated cardiometabolic parameters in lean AI women versus lean non-AI women were examined by comparing HTG content and the markers of cardiometabolic risk between lean AI women and non-AI women. Similar to the ethnic differences that we observed in lean AI men versus lean non-AI men (Table 3), we found that lean AI women had an approximately 1.5-fold increase in HTG as well as increased IMCL compared with lean non-AI women, which was associated with increased plasma insulin, triglyceride, and LDL-cholesterol concentrations and IR, as reflected by increased HOMA-IR and decreased ISI (Supplemental Table 3).

Given the observed sex-based reductions in HTG, IMCL, and IR in women (both AI and nonAI) compared with men (both AI and non-AI), we examined whether these protective effects wane with menopause. We found that HTG was 2.5-fold higher in postmenopausal women compared with premenopausal women and that this increase in HTG was associated with increased IMCL, EMCL, fasting plasma glucose, triglyceride, and total cholesterol, due mostly to increased LDL-cholesterol concentrations, and increased systolic and diastolic blood pressure (Table 4).

\section{Discussion}

The current upper normal level of HTG is defined as $5.56 \%$ based upon a single study in 345 lean individuals where HTG content was measured by a whole-body ${ }^{1} \mathrm{H}$ MRS $1.5 \mathrm{~T}$ system (15). In this study we examined a larger group of approximately 1500 healthy, lean, nonsmoking volunteers, who were carefully screened to exclude confounding factors such as excessive alcohol intake, any organ or systemic medical conditions, and any medications other than oral contraceptives, and characterized each participant using ${ }^{1} \mathrm{H}$ MRS of liver, IMCL and EMCL content, laboratory tests, and an OGTT. We found that the 95th percentile of HTG content in healthy, lean individuals was only $1.85 \%$, which was approximately 3 -fold 


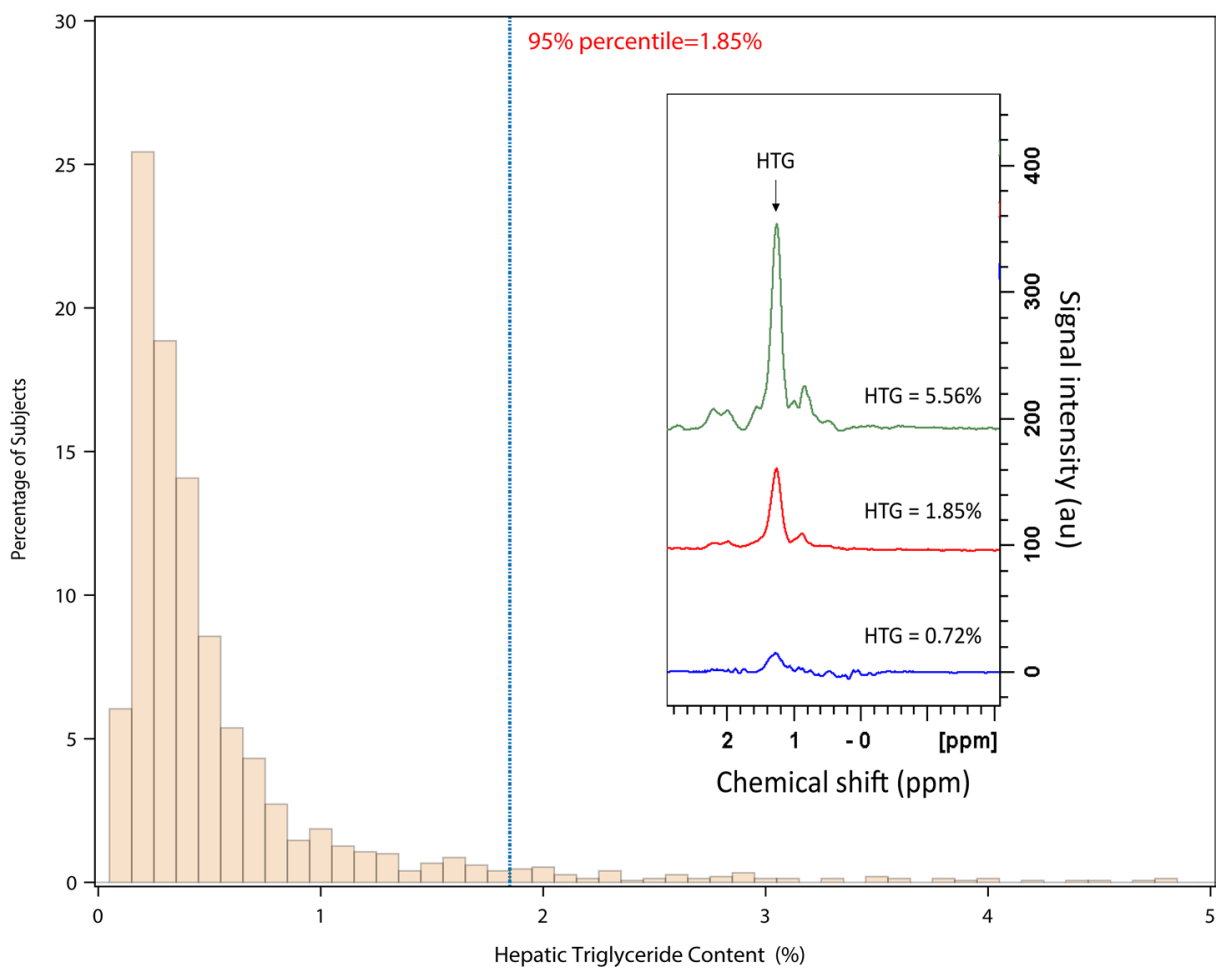

Figure 2. Distribution of HTC content in lean $\left(B M I<25 \mathrm{~kg} / \mathrm{m}^{2}\right)$ non-Al individuals $(\boldsymbol{n}=\mathbf{1 5 0 6})$. Consisting of $n=596$ men, $n=910$ women. Insert shows 3 typical ${ }^{1} \mathrm{H}$ MRS spectra of HTC obtained at 4 T. ppm, parts per million.

lower than the previously defined criterion for NAFL. The reason for this difference is unclear. It is possible that differences in the study inclusion criteria used by Szczepaniak et al. may account for these observed differences in HTG, because the group of 345 individuals studied is described only as being nonobese and nondiabetic, with normal liver function tests and minimal alcohol intake (15). Additional reasons for this discrepancy could be the large voxel size $\left(27 \mathrm{~cm}^{3}\right)$, used by Szczepaniak et al. in their studies, which potentially could include fat contamination from extrahepatic sites (e.g., subcutaneous fat, visceral fat, gallbladder, etc.), and the use of an assumed transverse relaxation time $\left(\mathrm{T}_{2}\right)$ for all of their participants, which may overestimate the signal areas in the ${ }^{1} \mathrm{H}$ MRS lipid spectrum.

To avoid these limitations, we assessed HTG content by ${ }^{1} \mathrm{H}$ MRS in 4 relatively small liver volumes $\left(15 \mathrm{~mm}^{3}\right)$, which were carefully located to avoid nearby abdominal and subcutaneous adipose tissue. Furthermore, to further ensure proper volume location, we gated and synchronized our ${ }^{1} \mathrm{H}$ MRS acquisition to the end of expiration to minimize the effects of movement during respiration. A water-suppressed lipid spectrum and a lipid-suppressed water spectrum were acquired in 4 different locations in each participant to account for liver inhomogeneity and the average value was used. In addition, the HTG content was corrected for $\mathrm{T}_{2}$ measured in each participant.

To determine whether HTG $\leq 1.85 \%$ would be meaningful as the revised cutoff for NAFL, we examined whether the group of non-AI individuals with HTG content $>1.85 \%$ and $\leq 5.56 \%$ were insulin resistant and whether they had any cardiometabolic related risk factors compared with individuals with HTG content $\leq 1.85 \%$. We found that HTG between $>1.85 \%$ and $\leq 5.56 \%$ was accompanied by increased cardiometabolic risk factors as reflected by increased blood pressure, fasting plasma insulin, triglycerides, total cholesterol, LDL, and uric acid, and reduced HDL concentrations and IR, compared with non-AI individuals with $\mathrm{HTG} \leq 1.85 \%$. Thus, our findings show that HTG content $>1.85 \%$ but less than the current definition of NAFL of HTG $>5.56 \%$ is strongly associated with whole-body IR and increased cardiometabolic risk factors and that NAFL reflects more than a benign accumulation of lipid in hepatocytes.

We next examined whether there are sex differences in HTG and associated cardiometabolic risk factors in this cohort of healthy, mostly lean, young non-AI individuals and found that the mean HTG content was approximately $33 \%$ lower in lean non-AI women versus lean non-AI men. Furthermore, this reduction in HTG in the non-AI women was associated with reduced systolic and diastolic blood 
Table 1. Participant characteristics and associated cardiometabolic parameters after a 12-hour fast in non-Al individuals with HTC content $\leq 1.85 \%$ versus non-Al individuals with HTG content $>1.85$ and $\leq 5.56 \%$

\begin{tabular}{|c|c|c|c|}
\hline Characteristic & HTG $\leq 1.85 \%(n=1731)$ & HTC $>1.85$ and $\leq 5.56 \%(n=119)$ & $P$ value $^{A}$ \\
\hline Female - no. (\%) & $1032(59.6 \%)$ & $46(38.7 \%)$ & $<0.0001^{\mathrm{B}}$ \\
\hline Age - y & $26.7 \pm 11.3$ & $37.3 \pm 17.7$ & $<0.0001^{\mathrm{C}}$ \\
\hline Systolic blood pressure - mmHg & $108.6 \pm 11.5$ & $115.9 \pm 10.7$ & 0.3106 \\
\hline Diastolic blood pressure $-\mathrm{mmHg}$ & $64.5 \pm 8.2$ & $69.7 \pm 8.0$ & 0.0156 \\
\hline Fasting plasma glucose $-\mathrm{mg} / \mathrm{dL}$ & $88.3 \pm 7.4$ & $92.7 \pm 7.6$ & 0.0931 \\
\hline Total cholesterol - mg/dL & $155.3 \pm 29.4$ & $177.6 \pm 34.4$ & 0.0001 \\
\hline LDL-cholesterol - mg/dL & $86.2 \pm 25.6$ & $107 \pm 33.5$ & $<0.0001$ \\
\hline HDL-cholesterol - mg/dL & $54 \pm 15.2$ & $48.1 \pm 15.2$ & 0.003 \\
\hline AST - U/L & $19.3 \pm 26.4$ & $20 \pm 9.4$ & 0.8704 \\
\hline$A L T-U / L$ & $15.4 \pm 14.5$ & $21.1 \pm 9.8$ & 0.0696 \\
\hline HTG - \% & $0.38(1.94)$ & $2.85(1.36)$ & $<0.0001$ \\
\hline IMCL - \% & $0.98 \pm 0.43$ & $1.23 \pm 0.53$ & 0.34 \\
\hline EMCL - \% & $1.05 \pm 0.74$ & $1.36 \pm 1.17$ & 0.8913 \\
\hline Body fat - \% & $21.9 \pm 8$ & $26.2 \pm 9$ & 0.0005 \\
\hline
\end{tabular}

pressure; lower fasting plasma concentrations of glucose, LDL-cholesterol, and uric acid; and increased plasma HDL-cholesterol concentrations. Applying this new criterion (HTG $\leq 1.85 \%$ ) for NAFL, we found that the prevalence of NAFL was $65 \%$ in overweight non-AI men, $52 \%$ in obese non-AI men, $35 \%$ in overweight non-AI women, and $48 \%$ in obese non-AI women.

Consistent with our prior studies, we found that healthy, lean AI men had an approximately $40 \%$ increase in HTG content and increased IMCL content compared with healthy, lean non-AI men (16). Furthermore, this increased HTG and IMCL content in the AI men was associated with increased fasting and 2-hour postprandial plasma glucose and insulin concentrations, increased $\mathrm{HbA} 1 \mathrm{c}$, as well as decreased plasma triglyceride and HDL-cholesterol concentrations and whole-body IR as reflected by increased HOMA-IR and decreased ISI. The cause of this difference is still unknown. However, we have shown that a modest reduction in body weight in this group of men was sufficient to significantly reduce HTG content from $14.0 \%$ to $3.8 \%(P=0.05)(21)$ and improve the NAFL-associated dyslipidemia and IR, indicating that body weight is left-shifted in this ethnic group and that their BMI in general should be below $25 \mathrm{~kg} / \mathrm{m}^{2}$, which currently defines leanness in non-AI (22). However, for comparison we still have applied the current definition of normal BMI $<25 \mathrm{~kg} / \mathrm{m}^{2}$ to both groups and matched them for BMI.

We also examined whether there are sex differences in HTG content and cardiometabolic parameters in AI men versus AI women. Similar to the sex differences that we observed in non-AI individuals, we observed an approximately 50\% reduction in HTG in lean AI women compared with lean AI men, and this reduction in HTG content in the AI women was associated with lower fasting and postprandial glucose concentrations, uric acid, AST, and ALT and increased plasma concentrations of HDL-cholesterol.

We next examined whether there were similar ethnic differences in HTG and these associated cardiometabolic parameters in AI women versus non-AI women. Similar to the ethnic differences that we observed in lean AI men versus lean non-AI men, we found that lean AI women had an approximately 2-fold increase in HTG content compared with lean non-AI women, and this increased HTG content was associated with increased plasma insulin, triglyceride, and LDL concentrations and IR as reflected by increased HOMA-IR and decreased ISI. Applying the criterion for NAFL of HTG $\leq 1.85 \%$, we found that 
Table 2. Sex differences in HTG content and associated cardiometabolic parameters in non-AI men versus non-AI women

\begin{tabular}{|c|c|c|c|}
\hline Characteristic & $\begin{array}{c}\text { Non-AI men } \\
(n=806)\end{array}$ & $\begin{array}{c}\text { Non-Al women } \\
(n=1091)\end{array}$ & $P$ value $^{A}$ \\
\hline Age - y & $28 \pm 13$ & $28 \pm 12$ & $<0.0001^{\mathrm{B}}$ \\
\hline Systolic blood pressure - mmHg & $114.7 \pm 10.3$ & $105.3 \pm 10.9$ & $<0.0001$ \\
\hline Diastolic blood pressure - $\mathrm{mmHg}$ & $67.2 \pm 8.3$ & $63.4 \pm 8.0$ & $<0.0001$ \\
\hline Fasting plasma glucose - mg/dL & $91.1 \pm 7.4$ & $87.2 \pm 8.4$ & $<0.0001$ \\
\hline Triglyceride $-\mathrm{mg} / \mathrm{dL}$ & $79.3 \pm 47.4$ & $74.7 \pm 51.9$ & 0.8527 \\
\hline Total cholesterol - mg/dL & $155.3 \pm 31.3$ & $158.6 \pm 30.1$ & 0.0002 \\
\hline $\mathrm{LDL}-\mathrm{mg} / \mathrm{dL}$ & $91.1 \pm 27.9$ & $85.6 \pm 26.1$ & 0.0199 \\
\hline $\mathrm{HDL}-\mathrm{mg} / \mathrm{dL}$ & $47.9 \pm 13.1$ & $57.4 \pm 15.6$ & $<0.0001$ \\
\hline AST - U/L & $21.4 \pm 32.1$ & $18 \pm 19$ & 0.0295 \\
\hline HOMA-IR & $2.24 \pm 1.65$ & $2.08 \pm 1.31$ & 0.4391 \\
\hline $\mathrm{ISI}-\mathrm{dL} / \mathrm{min}$ per $\mu \mathrm{U} / \mathrm{mL}$ & $4.8 \pm 2.5$ & $4.9 \pm 2.4$ & 0.427 \\
\hline HTC content - \% & $0.58(2.83)$ & $0.39(2.30)$ & $<0.0001$ \\
\hline IMCL - \% & $1.03 \pm 0.48$ & $1.00 \pm 0.45$ & 0.1672 \\
\hline EMCL - \% & $1.03 \pm 0.83$ & $1.13 \pm 0.75$ & $<0.0001$ \\
\hline Body fat - \% & $16.1 \pm 5.8$ & $27 \pm 6.5$ & $<0.0001$ \\
\hline
\end{tabular}

Values with " \pm " are means \pm SD except for HTG content, for which geometric mean and SD were used. ${ }^{A} P$ value adjusted for age and BMI unless otherwise specified. ${ }^{B}$ Simple $t$ test. ${ }^{C} \mathrm{BMI}$ is the weight in kilograms divided by the square of the height in meters. ${ }^{D}$ Adjusted for age. HbA1c, hemoglobin A1c.

the prevalence of NAFL was $78 \%$ in lean AI men, $85 \%$ in overweight $\mathrm{AI}$ men, $86 \%$ in obese $\mathrm{AI}$ men, $22 \%$ in lean $\mathrm{AI}$ women, and $15 \%$ in overweight $\mathrm{AI}$ women.

We further examined whether these protective effects in women decrease with menopause and found that HTG was 2.5-fold higher in postmenopausal women compared with premenopausal women and that this increase in HTG was associated with increased cardiometabolic risk factors (increased fasting plasma glucose, insulin, triglyceride, and total cholesterol, due mostly to increased LDL concentrations, and increased systolic and diastolic blood pressure).

Taken together these results demonstrate both an ethnic and sex effect on HTG content and associated cardiometabolic risk factors where both AI men and AI women are prone to higher HTG content and increased IR and associated cardiometabolic risk factors than their respective non-AI sex and that the female sex promotes reduced HTG content and reductions in associated cardiometabolic risk factors.

The effects of sex on HTG content are controversial, with some studies showing lower prevalence of NAFL in women than men $(15,17,22-26)$ but other studies showing increased prevalence of NAFL in women (27-29). In the Dallas Heart Study, Browning et al. observed higher HTG content in men than women, which they attributed to increased alcohol consumption in men (17). In this study, we found that women (both AI and non-AI) had decreased HTG content compared with men (both AI and nonAI). However, this reduction in HTG in women was independent of alcohol consumption, suggesting an important sex-related hormonal contribution to this phenomenon related to menopause. Consistent with this hypothesis, we found that HTG was 2.5-fold higher in postmenopausal women compared with premenopausal women and that this increase in HTG content was associated with increased IMCL and EMCL content and cardiometabolic risk factors. These data are consistent with rodent studies demonstrating that estrogen treatment in ovariectomized mice has a protective effect from developing high-fat diet-induced NAFL $(30,31)$.

In summary, here we show that the $95 \%$ upper limit for HTG content in young, lean, non-AI individuals was $1.85 \%$ and that HTG content above this value was associated with increased whole-body IR and 
Table 3. Ethnic differences in HTG content and associated cardiometabolic parameters in lean (BMI $\left.<25 \mathrm{~kg} / \mathrm{m}^{2}\right)$ non-Al men versus lean Al men

\begin{tabular}{|c|c|c|c|}
\hline Characteristic & $\begin{array}{c}\text { Non-AI men } \\
(n=596)\end{array}$ & $\begin{array}{c}\text { Al men } \\
(n=157)\end{array}$ & $P$ value $^{A}$ \\
\hline Age $-y$ & $27 \pm 11$ & $28 \pm 11$ & 0.19 \\
\hline Systolic blood pressure - mmHg & $113.7 \pm 9.9$ & $112.3 \pm 10.1$ & 0.15 \\
\hline Diastolic blood pressure $-\mathrm{mmHg}$ & $66.4 \pm 7.7$ & $66.8 \pm 7.7$ & 0.55 \\
\hline Fasting plasma glucose $-\mathrm{mg} / \mathrm{dL}$ & $90.1 \pm 7.3$ & $94.2 \pm 11.8$ & $<0.0001$ \\
\hline Triglyceride $-\mathrm{mg} / \mathrm{dL}$ & $74.3 \pm 40.2$ & $92 \pm 54.1$ & $<0.0001$ \\
\hline Total cholesterol - mg/dL & $152.2 \pm 29.6$ & $153.1 \pm 25.3$ & 0.86 \\
\hline $\mathrm{LDL}-\mathrm{mg} / \mathrm{dL}$ & $88.3 \pm 26.7$ & $92.8 \pm 24.3$ & 0.08 \\
\hline $\mathrm{HDL}-\mathrm{mg} / \mathrm{dL}$ & $48.6 \pm 13.2$ & $45.5 \pm 10.9$ & 0.007 \\
\hline AST - U/L & $21 \pm 36.1$ & $20.5 \pm 6.6$ & 0.88 \\
\hline HOMA-IR & $2.05 \pm 1.12$ & $2.56 \pm 1.48$ & $<0.0001$ \\
\hline $\mathrm{ISI}-\mathrm{dL} / \mathrm{min}$ per $\mu \mathrm{U} / \mathrm{mL}$ & $5.0 \pm 2.6$ & $3.9 \pm 2.3$ & $<0.0001$ \\
\hline HTC content - \% & $0.48(2.36)$ & $0.76(2.86)$ & $<0.0001$ \\
\hline IMCL content - \% & $0.95 \pm 0.41$ & $1.1 \pm 0.5$ & $<0.0001$ \\
\hline EMCL content - \% & $0.96 \pm 0.80$ & $1.2 \pm 0.9$ & 0.001 \\
\hline Body fat - \% & $14.3 \pm 4.7$ & $17.1 \pm 5.3$ & $<0.0001$ \\
\hline
\end{tabular}

Values with " \pm " are means \pm SD except for HTC content, for which geometric mean and SD were used. ${ }^{A} P$ value adjusted for age and BMI except for variable age and BMI. ${ }^{\mathrm{B}} \mathrm{BMI}$ is the weight in kilograms divided by the square of the height in meters.

increased cardiometabolic risk factors. Given the key role for NAFL and IR in the development of cardiometabolic disease as well as obesity-associated cancers, these results suggest that it will be important to develop new therapies in addition to caloric restriction $(14,21)$ for NAFL/NASH to achieve reductions in HTG content below $1.85 \%$ to reverse IR and/or prevent cardiometabolic events. Furthermore, we found significant sex and ethnic differences in HTG content in healthy, young, lean individuals. Understanding these associations between sex and ethnicity with NAFL will allow precision medicine to target specific groups to improve health, prevent disease, and reduce the rates of morbidity and mortality associated with NAFL and its associated pathologies (32).

\section{Methods}

Human participants. From 2006 to 2020 we advertised in the local community and after an initial phone screen prospectively recruited and studied 2331 (1088 men and 1243 women) healthy, nonsmoking, sedentary individuals (not participating in regular physical activity, with a sedentary lifestyle, and on average walking $<10,000$ steps daily); with minimal history of alcohol consumption (less than 15 grams per day); and taking no medications (Figure 1).

Based upon our previous studies showing a higher prevalence of IR and hepatic lipid content in healthy, normal-weight AI men as compared with healthy, normal-weight eastern Asian, Black, White, and Hispanic individuals (16), each group were divided into non-AI individuals $(n=1897)$ and AI individuals $(n=434)$, all of whom were mostly between the ages of 25 to 35 years and lean. Ethnicity was self-reported (33). Each participant answered a questionnaire (34) about usual daily intake of food, alcohol use, eating habits, changes in body weight over the past 12 months, and physical activities. Habitual physical activity was measured over 3 consecutive days by a pedometer (Sportline, Inc.). The participants were also asked to describe the food and snacks consumed the day prior to the study visit, and the calorie count and composition of the diet for each participant were assessed. All participants underwent a complete medical history 
Table 4. Effects of menopause on HTC content and associated cardiometabolic parameters

\begin{tabular}{|c|c|c|c|}
\hline Characteristic & $\begin{array}{l}\text { Premenopausal women } \\
\qquad(n=1143)\end{array}$ & $\begin{array}{l}\text { Postmenopausal women } \\
\qquad(n=100)\end{array}$ & $P$ value $^{A}$ \\
\hline Age $-y$ & $24.8 \pm 6.3$ & $61.7 \pm 9.8$ & $<0.0001$ \\
\hline $\mathrm{BMI}-\mathrm{kg} / \mathrm{m}^{2}$ & $22.1 \pm 3.2$ & $24.6 \pm 3.4$ & $<0.0001$ \\
\hline Systolic blood pressure - mmHg & $104.2 \pm 10.0$ & $114 \pm 14.1$ & $<0.0001$ \\
\hline Diastolic blood pressure $-\mathrm{mmHg}$ & $62.9 \pm 7.7$ & $67.7 \pm 8.6$ & 0.0003 \\
\hline Fasting plasma glucose $-\mathrm{mg} / \mathrm{dL}$ & $86.9 \pm 8.6$ & $92.6 \pm 7.5$ & $<0.0001$ \\
\hline Triglyceride - mg/dL & $72.7 \pm 38.4$ & $107.2 \pm 118.3$ & $<0.0001$ \\
\hline Total cholesterol - mg/dL & $156.1 \pm 27.6$ & $187.3 \pm 34.9$ & $<0.0001$ \\
\hline $\mathrm{LDL}-\mathrm{mg} / \mathrm{dL}$ & $84.7 \pm 24.9$ & $102.8 \pm 29.5$ & $<0.0001$ \\
\hline $\mathrm{HDL}-\mathrm{mg} / \mathrm{dL}$ & $56.7 \pm 15.3$ & $60.6 \pm 18.3$ & 0.0006 \\
\hline AST - U/L & $17.7 \pm 18.3$ & $20.1 \pm 7.9$ & 0.24 \\
\hline HOMA-IR & $2.13 \pm 1.42$ & $2.33 \pm 1.64$ & 0.28 \\
\hline $\mathrm{ISI}$ - dL/min per $\mu \mathrm{U} / \mathrm{mL}$ & $4.8 \pm 2.5$ & $4.5 \pm 2.5$ & 0.72 \\
\hline Liver triglyceride content - \% & $0.39(2.19)$ & $1.02(3.21)$ & $<0.0001$ \\
\hline IMCL content - \% & $1.0 \pm 0.6$ & $1.4 \pm 0.7$ & $<0.0001$ \\
\hline EMCL content - \% & $1.1 \pm 0.7$ & $2.2 \pm 1.2$ & $<0.0001$ \\
\hline Body fat - \% & $26.7 \pm 6.4$ & $33.7 \pm 6.0$ & $<0.0001$ \\
\hline
\end{tabular}

Values with " \pm " are means \pm SD except for HTG content, for which geometric mean and SD were used. ${ }^{A} P$ value adjusted for BMI except for BMI itself and age. ${ }^{B} \mathrm{BMI}$ is the weight in kilograms divided by the square of the height in meters.

and physical examination, including measurement of $\mathrm{HbA1c}$, to confirm that they were not diabetic. Blood pressure was measured using a Welch Allyn Vital Signs Monitor 300 Series in the supine position. A 3-hour OGTT (75 grams) was performed along with plasma insulin concentrations, which were measured at -10 , $-5,0,10,20,30,60,90,120$, and 180 minutes during the OGTT, to calculate HOMA-IR (35) and the ISI (20) as indices of whole-body insulin sensitivity.

Analytical methods. Plasma glucose concentrations were measured using a YSI 2700 STAT Analyzer. Plasma concentrations of insulin were measured using a double-antibody radioimmunoassay kit (Linco). Plasma triglyceride, total cholesterol, HDL-cholesterol, LDL-cholesterol, uric acid, AST, and ALT concentrations were measured enzymatically (Cobas Mira Plus, Roche Diagnostics Corp.).

${ }^{1} H$ MRS assessment of hepatic, intramyocellular, and extramyocellular lipid content. HTG, IMCL, and EMCL contents were quantified by ${ }^{1} \mathrm{H}$ MRS at $4 \mathrm{~T}$ (Bruker) as previously described (35). Briefly, HTG was measured by ${ }^{1} \mathrm{H}$ respiratory cycle-gated STEAM spectroscopy in a $15 \times 15 \times 15 \mathrm{~mm}^{3}$ voxel. The acquisition was synchronized to the respiratory cycle and triggered at the end of expiration. A water-suppressed lipid spectrum and a lipid-suppressed water spectrum were acquired in 4 different locations to account for liver inhomogeneity and the average value was used. In addition, in each participant, HTG content was corrected for $\mathrm{T}_{2}$, using the transverse relaxation times of $22 \mathrm{~ms}$ for water and $44 \mathrm{~ms}$ for lipid. In each individual with a total HTG content above $4 \%$, we measured $\mathrm{T}_{2}$ and used this correction to calculate the final HTG content (35). IMCL and EMCL contents were measured in the soleus muscle by ${ }^{1} \mathrm{H}$ STEAM spectroscopy in a 10 $\times 10 \times 10 \mathrm{~mm}^{3}$ voxel using an $8.5 \mathrm{~cm}$ diameter circular ${ }^{13} \mathrm{C}$ surface coil with twin, orthogonal, circular, 13 $\mathrm{cm}{ }^{1} \mathrm{H}$ quadrature coils as described (35). Scout images of the lower leg were obtained to ensure correct positioning and to define an adequate volume for localized shimming using the FASTMAP procedure (35).

Calculations. The ISI was calculated from the plasma glucose and insulin concentrations before and during the OGTT as described (20). The ISI represents the composite whole-body insulin sensitivity, reflecting both hepatic and peripheral tissue insulin sensitivity. HOMA-IR was calculated according to this formula (36): (fasting plasma insulin $[\mu \mathrm{U} / \mathrm{L}] \times$ fasting plasma glucose $[\mathrm{mg} / \mathrm{dL}]) /(22.5 \times 18)$. 
Statistics. Variables are presented as mean \pm SD or frequency and percentage as appropriate. The distribution of HTG in a subset of 1506 lean non-AI individuals was examined to obtain the 95th percentile as the cutoff of the normal value. A larger non-AI subset with the entire BMI range was classified as either HTG $\leq 1.85 \%$ or $>1.85 \%$ and $\leq 5.56 \%$. Patient characteristics and 12 -hour fasting cardiometabolic parameters were compared using general linear regression adjusting for age, BMI, and sex. Similar multivariate linear regression analyses were performed to examine group differences by sex, ethnic group, and age of women. Since HTG was right skewed, log transformation was conducted for group comparisons, and geometric mean and SD are presented. Statistical significance was set at $P<0.05,2$ sided, and all analyses were performed using SAS 9.4.

Study approval. Written informed consent was obtained from each participant. The study protocol conformed to the ethical guidelines of the 1975 Declaration of Helsinki as reflected in a priori approval by the Yale Institutional Review Board.

\section{Author contributions}

KFP and GIS designed the experiments and wrote the manuscript with assistance from SD, FL, and DLR. KFP and SD performed the studies. FL performed all the statistical analyses. KFP, SD, FL, DLR, and GIS obtained and interpreted data.

\section{Acknowledgments}

The authors would like to thank Anne Impellizeri, MS; Irina Smolgovsky; Mikhail Smolgovsky; Gina Solomon, RN; Catherine Parmelee, RN; Johanna J'Son-Toft; and the Yale Center for Clinical Investigation-Yale/New Haven Hospital Research Unit for technical assistance. The research was supported by grants from the United States Department of Health and Human Resources (NIH/National Institute of Diabetes and Digestive and Kidney Diseases): R01 DK113984, P30 DK45735, U24 DK59635, and UL1 RR024139; and the Novo Nordisk Foundation (NNF18CC0034900).

Address correspondence to: Kitt F. Petersen or Gerald I. Shulman, Yale School of Medicine, PO Box 208020, New Haven, Connecticut 06520-8020 USA. Email: kitt.petersen@yale.edu (KFP). Email: gerald.shulman@yale.edu (GIS).

1. Jenkins DJA, et al. Glycemic index, glycemic load, and cardiovascular disease and mortality. NEngl J Med. 2021;384(14):1312-1322.

2. Kaneko H, et al. Lipid profile and subsequent cardiovascular disease among young adults aged < 50 years. Am J Cardiol. 2020;142:59-65

3. Lee $\mathrm{H}$, et al. Metabolic dysfunction-associated fatty liver disease and incident cardiovascular disease risk: a nationwide cohort study. Clin Gastroenterol Hepatol. 2020;19(10):2138-2147.

4. Friedman SL, et al. Mechanisms of NAFLD development and therapeutic strategies. Nat Med. 2018;24(7):908-922.

5. Samuel VT, Shulman GI. The pathogenesis of insulin resistance: integrating signaling pathways and substrate flux. J Clin Invest. 2016;126(1):12-22.

6. Kotronen A, et al. Increased liver fat, impaired insulin clearance, and hepatic and adipose tissue insulin resistance in type 2 diabetes. Gastroenterology. 2008;135(1):122-130.

7. Taylor R, et al. Remission of human type 2 diabetes requires decrease in liver and pancreas fat content but is dependent upon capacity for beta cell recovery. Cell Metab. 2018;28(4):667.

8. Noureddin M, Sanyal AJ. Pathogenesis of NASH: the impact of multiple pathways. Curr Hepatol Rep. 2018;17(4):350-360

9. Loomba R, et al. Nonalcoholic fatty liver disease progression rates to cirrhosis and progression of cirrhosis to decompensation and mortality: a real world analysis of Medicare data. Aliment Pharmacol Ther. 2020;51(11):1149-1159.

10. Mantovani A, et al. Non-alcoholic fatty liver disease and increased risk of incident extrahepatic cancers: a meta-analysis of observational cohort studies [published online March 8, 2021]. Gut. https://doi.org/10.1136/gutjnl-2021-324191.

11. Banini BA, Sanyal AJ. NAFLD-related HCC. Adv Cancer Res. 2021;149:143-169.

12. Huang DQ, et al. Global epidemiology of NAFLD-related HCC: trends, predictions, risk factors and prevention. Nat Rev Gastroenterol Hepatol. 2021;18(4):223-238.

13. Simon TG, et al. Cancer risk in patients with biopsy-confirmed nonalcoholic fatty liver disease: a population-based cohort study. Hepatology. 2021;74(5):2410-2423.

14. Petersen KF, et al. Reversal of nonalcoholic hepatic steatosis, hepatic insulin resistance, and hyperglycemia by moderate weight reduction in patients with type 2 diabetes. Diabetes. 2005;54(3):603-608.

15. Szczepaniak LS, et al. Magnetic resonance spectroscopy to measure hepatic triglyceride content: prevalence of hepatic steatosis in the general population. Am J Physiol Endocrinol Metab. 2005;288(2):E462-E468.

16. Petersen KF, et al. Increased prevalence of insulin resistance and nonalcoholic fatty liver disease in Asian-Indian men. Proc Natl Acad Sci U S A. 2006;103(48):18273-18277.

17. Browning JD, et al. Prevalence of hepatic steatosis in an urban population in the United States: impact of ethnicity. Hepatology. 2004;40(6):1387-1395. 
18. Petaja EM, Yki-Jarvinen H. Definitions of normal liver fat and the association of insulin sensitivity with acquired and genetic NAFLD-A systematic review. Int J Mol Sci. 2016;17(5):E633.

19. Kumashiro N, et al. Cellular mechanism of insulin resistance in nonalcoholic fatty liver disease. Proc Natl Acad Sci U S A. 2011;108(39):16381-16385.

20. Matsuda M, DeFronzo RA. Insulin sensitivity indices obtained from oral glucose tolerance testing: comparison with the euglycemic insulin clamp. Diabetes Care. 1999;22(9):1462-1470.

21. Petersen KF, et al. Apolipoprotein C3 gene variants in nonalcoholic fatty liver disease. $N$ Engl J Med. 2010;362(12):1082-1089.

22. Xu C, et al. Prevalence and risk factors for the development of nonalcoholic fatty liver disease in a nonobese Chinese population: the Zhejiang Zhenhai Study. Am J Gastroenterol. 2013;108(8):1299-1304.

23. Lazo M, et al. Prevalence of nonalcoholic fatty liver disease in the United States: the Third National Health and Nutrition Examination Survey, 1988-1994. Am J Epidemiol. 2013;178(1):38-45.

24. Wang Z, et al. Sex-specific prevalence of fatty liver disease and associated metabolic factors in Wuhan, south central China. Eur J Gastroenterol Hepatol. 2014;26(9):1015-1021.

25. Park SH, et al. Prevalence and risk factors of non-alcoholic fatty liver disease among Korean adults. J Gastroenterol Hepatol. 2006;21(1 pt 1):138-143.

26. Babusik P, et al. Nonalcoholic fatty liver disease of two ethnic groups in Kuwait: comparison of prevalence and risk factors. Med Princ Pract. 2012;21(1):56-62.

27. Bedogni G, et al. Incidence and natural course of fatty liver in the general population: the Dionysos study. Hepatology. 2007;46(5):1387-1391.

28. Ayonrinde OT, et al. Sex-specific differences in adipose distribution and adipocytokines influence adolescent nonalcoholic fatty liver disease. Hepatology. 2011;53(3):800-809.

29. Summart U, et al. Sex differences in the prevalence of nonalcoholic fatty liver disease in the Northeast of Thailand: A population-based cross-sectional study. F1000Res. 2017;6:1630.

30. Camporez JP, et al. Cellular mechanism by which estradiol protects female ovariectomized mice from high-fat diet-induced hepatic and muscle insulin resistance. Endocrinology. 2013;154(3):1021-1028.

31. Camporez JP, et al. Anti-inflammatory effects of oestrogen mediate the sexual dimorphic response to lipid-induced insulin resistance. J Physiol. 2019;597(15):3885-3903.

32. Samuel VT, Shulman GI. Nonalcoholic fatty liver disease as a nexus of metabolic and hepatic diseases. Cell Metab. 2018;27(1):22-41

33. Flanagin A, et al. Updated guidance on the reporting of race and ethnicity in medical and science journals. JAMA. 2021;326(7):621-627.

34. Baecke JA, et al. A short questionnaire for the measurement of habitual physical activity in epidemiological studies. Am J Clin Nutr. 1982;36(5):936-942.

35. Rabøl R, et al. Reversal of muscle insulin resistance with exercise reduces postprandial hepatic de novo lipogenesis in insulin resistant individuals. Proc Natl Acad Sci U S A. 2011;108(33):13705-13709.

36. Matthews DR, et al. Homeostasis model assessment: insulin resistance and beta-cell function from fasting plasma glucose and insulin concentrations in man. Diabetologia. 1985;28(7):412-419. 